\title{
Estado actual de los postes
}

\author{
Artí́culo DE REVISIÓN
}

\section{de fibra de vidrio}

\author{
Current state of fiberglass post
}

\section{Resumen}

En la reconstrucción de dientes tratados endodónticamente tenemos muchas posibilidades clínicas y esto se debe al avance de los materiales y las técnicas. Es labor del profesional estar capacitado para realizar los procedimientos actuales modificando sus protocolos. Los materiales más comúnmente utilizados para la cementación de postes son los ionómeros de vidrio, los ionómeros de vidrio modificados con resina y los cementos de resina. Y para todos estos materiales necesitamos obtener una capa homogénea, delgada y carente de fisuras o burbujas. Estas características son difíciles de lograr sobre todo en el conducto radicular por las características del mismo. Se presenta una revisión de literatura sobre los materiales y técnicas más utilizados en la cementación de los postes prefabricados y se propone un protocolo que puede orientar al clínico para la obtención de resultados predecibles y confiables en el tiempo. Palabras clave: Técnica de perno munón, resinas compuestas, conducto radicular.

\section{Abstract}

In the reconstruction of endodontically treated teeth we have many clinical possibilities and this is due to the advancement of materials and technologies. The job of the professional is to be qualified to accomplish the current procedures modifying its protocols. The most commonly materials used for cementing posts are glass ionomer, resin modified glass ionomer and resin cements. For all these materials we need to obtain a homogeneous, thin layer without cracks or bubbles. These characteristics are difficult to achieve because of especial characteristics of root canal. A review of literature on the materials and techniques commonly used in the cementation of prefabricated post is presented and a protocol that can guide the clinician to obtain predictable and reliable results over time is proposed.
\end{abstract}

Keywords: Post and core technique; composites; root canal.

\begin{abstract}
César Lamas Lara1, Sergio AlvaradoMenacho², Liliana Terán-Casafranca², Giselle Angulo de la Vega ${ }^{3}$, Jesusa Jiménez Castro ${ }^{4}$, Ana Cisneros Cotrina ${ }^{4}$, Danny Pachas Amaringo 4 , Kelly Herrera Mejía ${ }^{4}$, Jerson Romero Llamoga ${ }^{4}$
\end{abstract}

1. Facultad de Odontología de la Universidad Particular San Martín de Porres, filial Norte, Perú.

2. Departamento Académico de Estomatología Rehabilitadora de la Facultad de Odontología de la Universidad Nacional Mayor de San Marcos, Perú.

3. Segunda Especialidad de Rehabilitación Oral de la Unidad de Postgrado de la Facultad de Odontología de la Universidad Nacional Mayor de San Marcos, Perú.

4. Escuela de Pregrado de Odontología de la Universidad Particular San Martín de Porres, filial Norte, Perú.

Correspondencia:

CD César Lamas Lara. Santa Honorata 415. Urb. Pando, 3era Etapa. Lima 1. Perú

Correo electrónico: cesar2579@hotmail.com

\section{Coautores}

Alvarado-Menacho: salvarado4@hotmail.com Teran-Casafranca: teranliliana@hotmail.com Ângulo: giseand@hotmail.com

Jiménez: jic 93@hotmail.com

Cisneros: ancico_923@hotmail.com

Pachas: daniel28_26@hotmail.com

Herrera: kellyestrella_92@hotmail.com

Romero: jerson-odonto@hotmail.com

Recibido: 25-08-15

Aceptado: 30-10-15

\section{Introducción}

En la actualidad existen 2 tipos de postes: los individualizados y los prefabricados. Muchos profesionales prefieren la utilización de los postes prefabricados en su práctica diaria por su practicidad y, en algunos casos, por ser menos agresivo con la estructura dental remanente. Estudios demuestran la eficacia de la utilización de los postes de fibra de vidrio pues reduce el riesgo de fractura radicular ${ }^{1,2}$. Un poste es considerando un retenedor intrarradicular cuyo objetivo fundamental es servir de anclaje para la reconstrucción del munón dentario perdido. Un poste, para ser considerado ideal, debe tener ciertas características como son: la forma debe ser similar al volumen del canal radicular presente, las propiedades mecánicas deben ser similares a las de la dentina, debe ser resistente para soportar las fuerzas masticatorias y su módulo de elasticidad debe ser lo más parecido a las estructuras histológicas que conforman el remanente dentario.

Todos los aditamentos protésicos fijos cuentan con dos tipos de retenciones: la retención primaria, que se logra por fricción, si hablamos de postes, cuan adaptado esté el poste a las paredes del conducto; y la retención secundaria, que la logramos a través de material de cementación; en el caso de los postes prefabricados en la mayoría de los casos no contamos con una buena retención primaria ya que este tipo de poste no sigue la anatomía del conducto radicular, valiéndonos solamente de la retención obtenida por el material de cementación (Fig.1).

El colocar un aditamento dentro del conducto no refuerza a la pieza den- taria, pero sí podría ayudar a disipar mejor las fuerzas ejercidas en ella, dependiendo del tipo de poste a colocar.

El gran aporte de los postes prefabricados de fibra se lo debemos a Duret, que introdujo los postes de fibra de carbono reforzados con resina en $1988^{3,4}$.

La interfase entre el poste y la dentina presenta condiciones desfavorables, ya que es difícil controlar que el cemento llegue correctamente a copiar los espacios del conducto radicular sin que se formen burbujas o irregularidades. Entonces, seleccionar el cemento más adecuado cobra vital importancia; en la actualidad los cementos de resina son considerados ideales en estas circunstancias al presentar mejores propiedades en relación con los cementos de ionómero de vidrio, por ejemplo ${ }^{5-7}$. 
Como agente cementante de los postes de fibra de vidrio generalmente se utiliza cementos de resina y estos funcionan a espesores entre $10 \mu$ y $30 \mu$, pero al utilizar postes con una forma predefinida, estos no van a seguir la anatomía del conducto radicular. Por tal motivo, el grosor del cemento de resina a utilizar va a ser mayor al ideal ${ }^{8}$ (Fig.2).

La preparación de la dentina radicular produce una capa de barrillo que debe ser eliminada antes de realizar los procesos adhesivos. Diversos irrigantes se han utilizado con la finalidad de facilitar la adhesión como son el $\mathrm{NaClO}$ al $5.25 \%$, clorhexidina al $2 \%$, ácido fosfórico, etc. ${ }^{9,10}$

La remoción del barrillo dentinario es un factor a tomar en cuenta cuando buscamos adhesión dentro del conducto radicular. Los irrigantes endodónticos influyen en la adhesión de los cementos resinosos a la dentina, ya que estos ocasionan una desmineralización y deproteinización de la dentina radicular facilitando la penetración de los tags de resina en los túbulos dentinarios ${ }^{11}$.

La retención de los adhesivos que requieren grabado ácido se basa en la hibridización de la dentina, entonces la aplicación del ácido grabador y su remoción completa, así como la aplicación del adhesivo, se torna compleja sobre todo en conductos estrechos. Entre las ventajas de utilizar cementos autoadhesivos para cementar postes destaca la simplicidad de su protocolo clínico, contribuyendo a la obtención de resultados predecibles ${ }^{12}$.

\section{Cementos resinosos autoadhesivos}

Los cementos autoadhesivos surgen como una alternativa en relación con los cementos resinosos convencionales. La gran ventaja que presentan estos cementos es su facilidad en la técnica volviendo sus resultados más predecibles. Su aplicación simplemente sería la mezcla de las pastas base y catalizadora, o la activación de las cápsulas; una vez completada la mezcla se aplica el material sobre la superficie a adherir. De esta manera se reducen los errores en la secuencia, propios de las técnicas anteriores; también la incompatibilidad entre los adhesivos autograbadores con los cementos resinosos quimiopolimerizables o de tipo dual ${ }^{14,15}$.

La adhesión de este tipo de cementos consta básicamente de una retención micromecánica y una interacción química entre los monómeros ácidos del cemento resinoso y la hidroxiapatita de la dentina. Supuestamente el cemento debería ser capaz de desmineralizar e infiltrar al mismo tiempo el sustrato dental ${ }^{16}$.

La polimerización de estos cementos se realiza tras la exposición a la luz o con un mecanismo de quimiopolimerización, ya que pertenecen al tipo de cementos duales. La reacción entre los grupos ácidos y el relleno alcalino asegura la neutralización de los monómeros ácidos. En las fases iniciales de su aplicación, producto de esta reacción ácido-base, se libera agua que debería favorecer al comportamiento hidrofílico del cemento, limitando la influencia de la humedad típica de la dentina. Posteriormente, el agua se comporta como un tapón para que el cemento desarrolle propiedades más hidrofóbicas y no incorpore agua resultante de la trasudación de los líquidos a través de los túbulos dentinarios ${ }^{16,17}$.

Al analizar el comportamiento de los cementos resinosos autoadhesivos, cabe destacar las diferencias entre dentina radicular y la dentina coronal. Las investigaciones concuerdan en señalar la ausencia de una capa híbrida y la limitada capacidad del cemento resinoso de desmineralizar la dentina. Si analizamos la fuerza de adhesión los valores de adhesión fueron similares a los cementos resinosos convencionales en el caso de postes prefabricados de fibra de vidrio ${ }^{18,19}$.

La mayor cantidad de fracasos de las restauraciones con postes de fibra se debe a fallas en la retención a nivel de la interfase dentina-cemento. De esto podemos deducir que la dentina radicular es un sustrato poco favorable para realizar procesos adhesivos ${ }^{20,21}$.

En relación a cómo tratar la superficie de los postes de fibra de vidrio antes de la cementación, se han propuesto diferentes tratamientos con la finalidad de aumentar la retención. Estos tratamientos pueden ser: químico (aplicación de agentes de enlace como el silano o sistemas adhesivos), mecánico (aplicación de ácido fosfórico o arenado con óxido de aluminio) y químico-mecánico (mezclando los procedimientos anteriores). Algunos postes vienen con tratamientos previos, por consiguiente no necesitan realizar estos procedimientos salvo si se han manipulado mucho y se requiere algún tipo de limpieza del mismo con alcohol o grabado ácido ${ }^{22,23}$.

\section{Colocación de postes de fibra de vidrio en} conductos amplios

Los conductos radiculares no presentan una forma circular precisa, generalmente presentan formas elípticas, como en el caso de caninos y premolares mandi- bulares. También se pueden presentar situaciones clínicas donde se ha preparado demasiado el conducto radicular (por la preparación biomecánica del tratamiento de conductos o por el retiro de un poste previo). Para estos casos podemos valernos de tres alternativas clínicas: La colocación de postes de fibra accesorios, adaptar el conducto radicular al poste a través de materiales que estén adheridos a la dentina radicular (como los ionómeros de vidrio) o adaptar el poste al conducto (a través de resina compuesta). También existiría la posibilidad de cementar el poste sin realizar ninguna adaptación previa, pero esto llevaría una mayor cantidad de volumen de cemento sobre todo en la parte cervical del conducto que podría llevar a fallas en esta interfase y por consiguiente su descementación (Fig.3, Fig.4).

\section{Postes de fibra de vidrio accesorios}

Consiste en colocar postes de fibra de vidrio accesorios dentro del conducto, además del poste principal con la finalidad de reducir la capa de cemento y por consiguiente se reduce la posibilidad de desalojo del mismo. Estudios demuestran que esta técnica distribuye mejor las fuerzas, distribuyéndolas hacia el ligamento periodontal en comparación con los postes únicos o los colados ${ }^{24}$.

\section{Reconstrucción de las paredes del con- ducto con ionómero de vidrio}

El objetivo de esta técnica es la reconstrucción de las paredes del conducto, básicamente en el tercio cervical utilizando ionómeros de alta viscosidad, con la finalidad de reducir el espesor de cemento y así prevenir la presencia de vacíos y burbujas dentro de la gruesa capa de cemento; estos espacios pueden propiciar una irregular distribución de fuerzas, una polimerización inadecuada por la presencia de oxígeno y una contracción de polimerización inadecuada por los espacios dentro del conducto ${ }^{25}$.

\section{Técnica del poste anatómico}

Se considera ideal preservar la mayor cantidad de estructura dentaria sana en la odontología actual y este concepto se traduce en desgastar lo menos posible las paredes del conducto radicular en la confección del poste; es decir, adaptar el poste al conducto y no el conducto al poste. Entonces debemos utilizar postes de poco diámetro para evitar desgastar dentina innecesariamente.

Lo ideal sería utilizar un poste que pueda copiar la anatomía del conducto radicular y que tenga un módulo de 
elasticidad similar a la dentina y esto se logra realizando la técnica del poste anatómico, descrita por el Dr. Simone Grandini y el Dr. Marco Ferrari de la Universidad de Siena ${ }^{2}$.

La técnica consiste básicamente en utilizar resina compuesta para realizar una impresión de conducto radicular obteniendo de esta manera una copia de su anatomía interna ${ }^{26,27}$, simulando la técnica del patrón de acrílico utilizada para postes colados. De esta manera, se obtendrá un poste de resina compuesta con un núcleo de fibra de vidrio (Fig.5, Fig.6).

La resina utilizada para el anatomizado del poste de fibra de vidrio sufre un proceso de contracción de polimerización (aunque este proceso requiere más investigaciones). Esto beneficiará el retiro del poste del conducto y creará además un espacio de fuga del cemento que evitará la presión hidráulica ${ }^{28}$.

La reducción del espesor de la capa de cemento disminuye la probabilidad de formación de burbujas o vacíos dentro del material (Fig.7). Estas áreas pueden desencadenar fisuras y disminuir la retención del poste. Otra manera de poder mejorar las condiciones desfavorables en la cementación de los postes es utilizar cementos adhesivos de lenta polimerización, porque se prolonga el tiempo, lo que aumenta las posibilidades de liberación del estrés de polimerización ${ }^{29,30}$.

La buena adaptación del poste de fibra de vidrio anatomizado aumenta la presión en el cemento de resina. La aplicación de presión suprime la porción acuosa y la formación de burbujas, dando como resultado un mejor contacto entre estos tres elementos (poste, cemento y dentina). Esto va a generar mayor retención por fricción y por consiguiente mayor resistencia adhesiva a la tracción. Algunos estudios sugieren que el aumento de la resistencia adhesiva está ligado a la retención por fricción, más que a la disminución del espesor de la capa de cemento ${ }^{30,31}$.

\section{Inyección del cemento dentro del conduc- to radicular}

La aplicación del cemento de resina dentro del conducto radicular es un factor importante a tomar en cuenta al momento de realizar la cementación de los mismos. A diferencia de los ionómeros de vidrio con los cuales podemos utilizar un léntulo garantizando de esta manera la eliminación de las burbujas de aire, los cementos resinosos no permiten su utilización ya que el movi- miento generado acelera el proceso de polimerización.

Una de las opciones que podríamos realizar sería embadurnar el poste con el cemento de resina dual y de esta manera realizar la cementación (Fig.8), pero este método no nos garantiza la no formación de burbujas de aire en esta interfase (Fig.9).

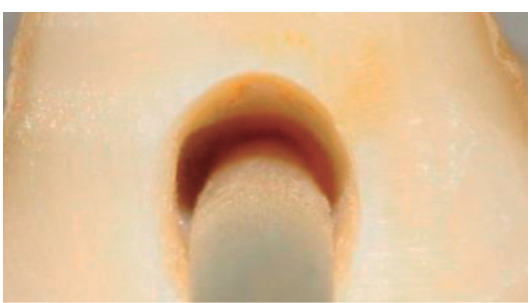

Fig. 1. Adaptación de un poste de fibra de vidrio dentro del conducto radicular.

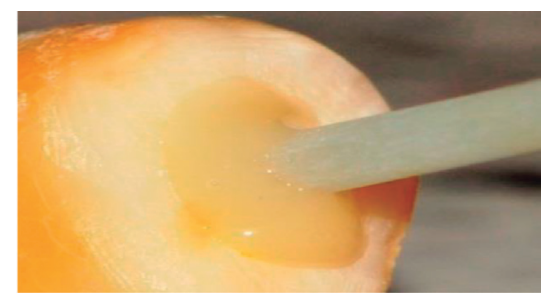

Fig. 2. Cementación de un poste de fibra de vidrio con cemento de resina dual

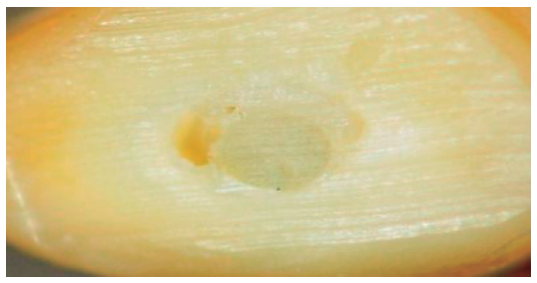

Fig. 3. Poste de fibra de vidrio cementado de manera convencional: Formación de burbujas de aire en la interfase de cementación.

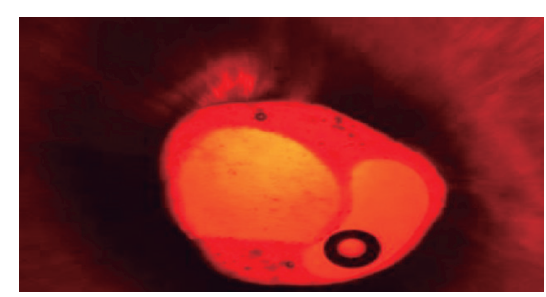

Fig. 4. Vista al microscopio de la interfase dentina-poste: Nótese la gran desadaptación del poste al conducto radicular y la formación de burbujas de aire en dicha interfase.

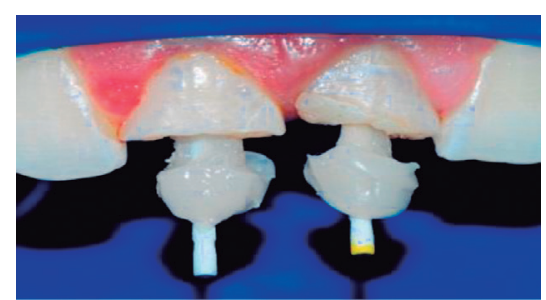

Fig. 5. Confección de postes anatómicos en piezas 1.1 y 2.1 , tomado de la Revista Increscendo; El poste anatómico en la reconstrucción de piezas dentarias anteriores 2014; 5(2): 2013.

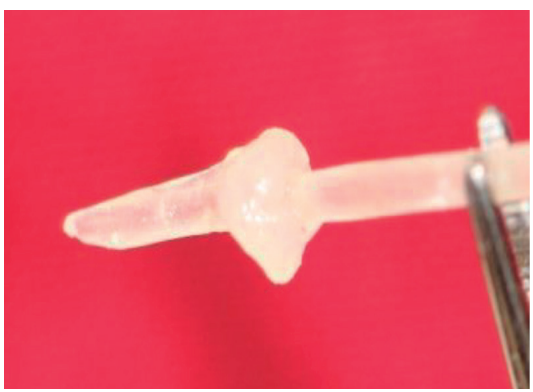

Fig. 6. Poste de fibra de vidrio anatomizado con resina compuesta, tomado de la Revista KIRU; 2014;11(1):83.

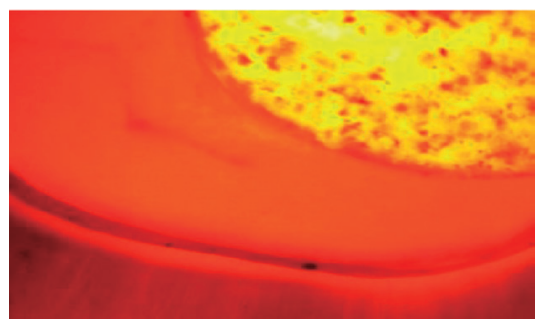

Fig. 7. Vista al microscopio de la interfase poste anatómico-dentina. Nótese la casi ausencia de irregularidades en la interfase.

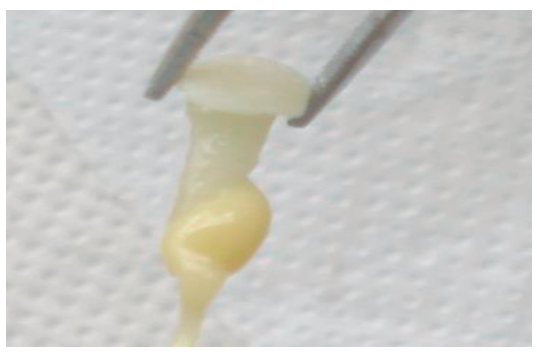

Fig. 8. Poste anatomizado embadurnado con cemento de resina dual.

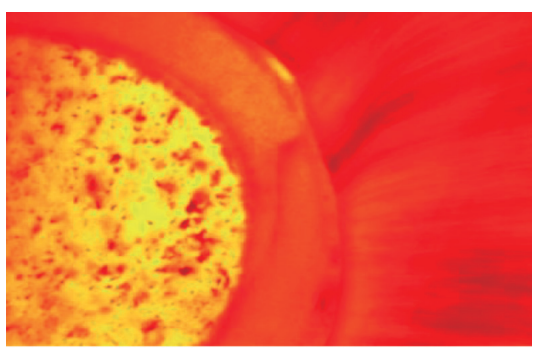

Fig. 9. Vista al microscopio de la interfase de cementación de un poste de fibra de vidrio anatomizado en donde se puede apreciar la formación de burbujas de aire.

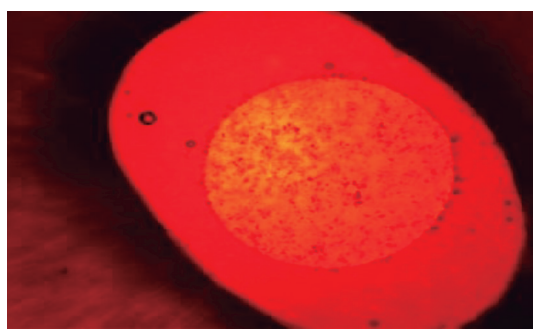

Fig. 10. Vista al microscopio de la cementación de un poste de fibra de vidrio al cual se inyectó el cemento de resina dual dentro del conducto. 
Algunas marcas de cementos de resina dual vienen con aplicadores muy delgados que nos facilitan la aplicación del cemento directamente dentro del conducto, reduciendo así la formación de burbujas de aire (Fig.10). Una manera práctica de inyectar el cemento de resina dentro del conducto (si no contamos con estos aplicadores) es cargar el cemento en un jeringa de tuberculina y de esta manera inyectarlo; aplicarlo de esta manera también reduce la formación de burbujas obteniendo una interfase homogénea.

\section{Propuesta para la cementación de postes de fibra de vidrio}

Luego de hacer nuestra revisión bibliográfica podemos plantear un protocolo para la cementación de los postes de fibra de vidrio:

1. Evaluar la morfología interna del sistema de conductos. Partiendo del principio que el endodoncista (o el que realizó la endodoncia en este caso) es el que mejor conoce la anatomía interna del conducto radicular es él quien debe realizar la desobturación.

2. Desobturación conservadora. Tratando de ser lo más conservador posible para evitar debilitar las paredes del conducto radicular. Siempre se debe utilizar 2 fresas: una desobturadora (para el retiro del material endodóntico dentro del conducto) y una conformadora para que el poste pueda ingresar correctamente, $y$ lograr una adaptación a nivel apical del poste.

3. Elección del diámetro del poste de fibra de vidrio. El poste debe adaptarse al conducto y no el conducto al poste; la mayoría de postes que utilizamos actualmente son cilindrocónicos, por consiguiente no vamos a lograr una adaptación del mismo en todo el conducto radicular; como mencionamos anteriormente la adaptación del poste debe ser a nivel apical del mismo, lo restante se puede anatomizar.

4. Evaluar la desadaptación del poste de fibra de vidrio a la porción cervical y media del conducto radicular. Esta evaluación puede realizarse visualmente, así como con la ayuda de una radiografía.

5. Anatomización del poste de fibra de vidrio. Si el caso lo requiere para reducir la capa de cemento de resina a utilizar.
6. Inyección del cemento de resina dual dentro del conducto. Este proceso es importante ya que reduce notablemente la formación de burbujas de aire que pueden afectar la retención del poste.

7. Cementación propiamente dicha. Comprende el proceso de llevar el poste dentro del conducto y el retiro de los excesos de cemento. Por la complejidad de lograr una correcta adhesión dentro del conducto se recomendaría utilizar cementos de resina dual autoadhesivos.

\section{Anatomizado del poste de fibra de vidrio}

Como protocolo se tiende a dejar entre 4 y $5 \mathrm{~mm}$ de material endodóntico al momento de la desobturación del conducto para la confección de un poste; pero esta tendencia está cambiando notablemente y la tendencia actual es a no realizar un poste tan largo o inclusive en algunos casos a no colocar postes, ya que en muchos casos en vez de ayudar al refuerzo de la pieza dentaria la debilita.

Una vez realizada la desobturación se realiza la conformación del conducto. Este paso es controversial, porque muchas veces desgastamos innecesariamente estructura dentaria sana con la finalidad de poder hacer ingresar el poste; y este concepto debe cambiar, debemos buscar postes que se adapten al conducto y que tengan un ligero ajuste a nivel apical.

Se coloca glicerina o vaselina líquida dentro del conducto, con la finalidad de que sirva de aislante, para que la resina compuesta que vamos a utilizar no se adhiera al conducto.

Al poste de fibra de vidrio se le acondiciona con silano con la finalidad de permitir que la resina se adhiera de una mejor manera. Acá hay un tema controversial, ya que algunos autores recomiendan la aplicación de ácido fosfórico sobre la superficie del poste, pero hay estudios que demuestran que grabar el poste de fibra no mejora los niveles de adhesión.

Una vez aplicado el silano, se deja evaporar por un minuto y se coloca resina compuesta sobre el poste con la finalidad de llevarlo al conducto y copiar su morfología de una manera similar a la técnica del patrón de acrílico utilizado en postes colados.

Una vez llevado el poste dentro del conducto se inicia su fotopolimerización por 80 segundos; cabe resaltar que du- rante el proceso de fotopolimerización es importante retirar y poner el poste dentro del conducto para evitar que se quede atrapado.

Una vez verificada la correcta adaptación del poste anatomizado se procede a la limpieza del conducto, irrigando primero con $\mathrm{NaClO}$ al $5.25 \%$ y luego para neutralizar el $\mathrm{NaClO}$ se irriga con suero fisiológico. El exceso de humedad se puede eliminar utilizando conos de papel y la limpieza del poste se realiza con una gasa embebida en alcohol.

De esta manera, el poste y el conducto estarían listos para el proceso de cementación.

Por lo difícil de lograr un buen proceso adhesivo dentro del conducto radicular se puede emplear cementos autoadhesivos con la finalidad de simplificar la técnica. Se aplica el cemento de resina de curado dual autoadhesivo dentro del conducto inyectándolo con puntas dispensadoras especiales o con una jeringa y se lleva el poste dentro del conducto; se retiran los excesos y se fotopolimeriza por 60 segundos o se espera 5 minutos a que complete su polimerización.

\section{Discusión}

Diversas causas se han atribuido a la fractura de los dientes tratados endodónticamente, como son: la fuerza excesiva durante la condensación de la gutapercha, la corrosión del poste metálico o la preparación excesiva del espacio para el poste. Entre mayor sea la estructura dentaria perdida, es menor la resistencia estructural del diente y, por lo tanto, mayor el riesgo a la fractura ${ }^{32-35}$.

$\mathrm{Si}$ bien es cierto que los postes colados se han comportado de una manera efectiva a lo largo de su aplicación en odontología, también es cierto que cuando el poste es sometido a fuerzas excesivas puede fracturar la pieza dentaria. Estudios demuestran que las fallas producidas en estos casos por lo general no son reparables en este tipo de poste. a diferencia de los postes de fibra de vidrio cuya principal causa de fracasos es la descementación ${ }^{36-39}$.

Estudios in vitro confirman que los postes de fibra de vidrio producen menor índice de fracturas radiculares que los postes metálicos colados. Estudios in vivo mencionan ausencia de fractura radicular en dientes restaurados con postes de fibra de vidrio, debido a que presentan propiedades físicas similares a la dentina, lo que genera menor transferencia de estrés en las estructuras radiculares $^{40-45}$. 
Estudios demuestran la formación de un gel amorfo (barrillo dentinario) dentro del conducto de cierta impermeabilidad sobre la parte superior del armazón de colágeno expuesto; y esto ha sido atribuido al efecto combinado de desnaturalización y colapso de la capa de desecho residual de colágeno; esta capa podría evitar la formación de tags de resina del cemento reduciendo su retención. La aplicación breve de una solución de $\mathrm{NaClO}$ al 5.25\%, antes de la cementación, se ha sugerido para remover este gel ${ }^{46}$.

El confeccionar un poste anatómico mejora la adaptación del poste al conducto radicular y reduce el grosor de la capa de cemento a emplear para su fijación; al reducir esta capa se logra disminuir la probabilidad de formación de burbujas o vacíos que pueden disminuir la retención del poste. La contracción de polimerización del agente cementante puede ser reducida de forma adicional cuando se utiliza un cemento autoadhesivo de autopolimerización, ya que se prolonga en tiempo de gelación del mismo ${ }^{29,47-49}$.

\section{Conclusiones}

- Las técnicas y los materiales para la restauración de dientes tratados endodónticamente han evolucionado exponencialmente en los últimos años. Con el avance de las investigaciones, técnicas que se consideraban seguras han quedado en desuso; por tal motivo, la preparación e investigación por parte del profesional es muy importante para ofrecer procedimientos duraderos y con resultados previsibles en el tiempo.

- El anatomizar los postes nos garantizan un factor muy importante que tienen los postes colados que es la retención primaria (por fricción) aumentando de esta manera su retención y reduciendo la capa del agente de cementación a valores más ideales; por consiguiente, un comportamiento biomecánico ideal.

- La inyección del cemento de resina dentro del conducto, así como la anatomización del poste de fibra de vidrio con resina compuesta, mejora las características de los postes en relación con su retención y nos garantiza una interfase de cemento de resina homogénea y delgada ante una situación clínica difícil como es el conducto radicular.

- El utilizar cementos de resina autoadhesivos para la cementación de postes es una opción válida ya que nos ofrece protocolos más simples y resultados más predecibles.

\section{Referencias bibliográficas}

1. Gomes J, Kina S. Adhesión en prostodoncia fija. En: Henostroza HG. Adhesión en odontología res-

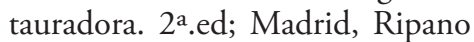
Editorial Médica; 2010. p. 367-95.

2. Ferrari M, Scotti R. Fibers Posts Characteristics and clinical applications. 1era ed. Roma: Ed Masson; 2002

3. Reeh E. Reduction in tooth stiffness as a result of endodontic and restorative procedures. J Endod. 1989;15(11):512-6

4. Sorensen J, Martinoff J. Intracoronal reinforcement and coronal converage. J. prost. Dent.1984; 51(6):780-4.

5. Schwartz RS, Robbins JW. Post placement and restoration of endodontically treated teeth: a literature review. J Endod 2004; 30(5):289-301.

6. Mezzomo E, Massa F, Libera SD. Fracture resistance of teeth restored with two different postand-core designs cemented with two different cements: an in vitro study. Part I. Quintessence Int 2003;34(4):301-6.

7. Dauvillier BS, Feilzer A, de Gee AJ, Davidson CL. Visco-elastic parameters of dental restorative materials during setting. J Dent Res 2000;79(3):818-23.

8. Shillingburg H, Hobo S, Whitsett L, Jacobi R, Brackett S. Fundamentos Esenciales en Prótesis Fija. 3ra ed. Madrid: Ed Quintessence; 2006.

9. Goldsmith M, Kishor M, Gulabivala. The effect of sodium hypochlorite irrigant concentration on tooth surface strain. J Endod 2002; 28(8): 575-9.

10. Coelho G, El-Mowafy O, Hernique J. Diametral tensile strength of a resin composite core with nonmetallic prefabricated posts: An in vitro study. J Prosth Dentist 2004; 91(4): 335-41.

11. Maki HY. Effect of endodontic irrigation on bonding of resin ce- ment to radicular dentin. Eur J Oral Sci 2005; 113(1): 70-6.

12. Bitter K, Meyer-Lueckel H, Priehn K, Kanjuparambil Jp, Neuman K, Kielbassa AM Effects of luting agent and thermocycling on bond strengths to root canal dentine. Int Endod J 2006;39(10) 809-18

13. Ferrari $\mathrm{M}$ y col. Bonding to root canal: Structural characteristics of the substrate, Am J Dent, 2000;13(5):255-60.

14. Tay FR, Pashley DH, Yiu CH, Sanares AM, Wei SH. Factors Contributing to the incompatibility between simplified-step adhesives and chemically-cured or dualcured composites. Part I. Singlestep self-etching adhesive. J Adhes Dent 2003; 5(1): 27-40.

15. Cheong C, King NM, Pashley DH, Ferrari M, Toledano M, Tay FR. Incompatibility of self-etch adhesives with chemical/dual cured composites: two-steps vs onestep systems. Oper Dent 2003; 28(6): 747-55.

16. Cedillo J, Espinoza R. Nuevas tendencias para la cementación de postes. ADM, 2011; 68(4): 196206

17. De Souza Costa CA, Hebling J, Randall RC, Human pulp response to resin cements used to bond inlay restorations. Dent Mater 2006; 22(10): 954-62.

18. Bitter K, Priehn K, Martus P, Kielbassa AM. In vitro evaluation of push-out bond strengths of various luting agents to tooth colored posts. J Prosthet Dent 2006; 95(4): 302-10.

19. Bateman GJ, Lloyd CH, Chadwick RG, Saunders WP. Retention of quartz-fibre-endodontic-posts with a self-adhesive dual cure resin cements. Eur J Prosthodont Restor Dent 2005; 13(1): 33-7.

20. Ferrari M, Vichi A, Mannocci F, Mason PN. Retrospective study of clinical performance of fiber posts. Am J Dent 2000;13(Spec No):9B13B.

21. Malferrari S, Monaco C, Scotti R. Clinical Evaluation of teeth restored with quartz fiber-reinforced epoxy resin posts. Int J Prosthodont 2003;16(1):39-44.

22. Magni E, Mazitelli C, Papacchini F, Radovic I, Goracci C, Coniglio 
I, Ferrari M. Adhesion between fiber posts and resin luting agents: a microtensile bond strength test and an SEM investigation following different treatment of the post surface. J Adhes Dent 2007;9(2):195-202.

23. Monticelli F, Toledano M, Tay FR, Cury AH, Goracci C, Ferrari M. Post surface conditioning improves interfacial adhesion in post/core restorations. Dent Mater 2006;22(7):602-9.

24. Maceri F, Martignoni M, Vairo G. Mechanical behaviour of endodontic restorations with multiple. J Biomech. 2007; 40(11):2386-98.

25. Cedillo J y col. Poste anatómico: reporte de un caso. RODYB, 2014;3(2):1-10

26. Lamas C, Jimenez J, Angulo G. Poste anatómico - reporte de caso. KIRU. 2014;11(1):81-5

27. Lamas C, Bobadilla C, Angulo G. El poste anatómico en la reconstrucción de piezas dentarias anteriores. In Cres, 2014;5(2):209-16.

28. Grandini S, Sapio S, Simonetti M. Use of anatomic post and core for reconstructing an endodontically treated tooth: a case report. J Adhes Dent. 2003;5(3):243-7.

29. Tay FR, Loushine RJ, Lambrechts P, Weller RN, Pashley DH. Geometric factors affecting dentin bonding in root canals: a theoretical modeling approach. J Endod, 2005;31(8): 584-9.

30. Chieffi N, Chersoni S, Papacchini F, Vano M. The effect of application sustained seating pressure on adhesive luting procedure. Dent Mater. 2007;23(2):159-66.

31. Faria-e-Silva AL, Pedrosa CF. Effect of relining on fiber post retention to root canal. J. Appl Oral Sci. 2009;17(6):600-4.
32. Sornkul E, Stannard JG. Strength of roots before and after endodontic treatment and restoration. J Endod 1992;18(9):440-3.

33. Lloyd PM, Palik JF. The philosophies of dowel diameter preparation: a literature review. J Prosthet Dent 1993;69(1):32-6.

34. Patel A, Gutteridge DL. An in vitro investigation of cast post and partial core design. J Dent 1996;24(4):281-7.

35. Marchi GM, Mitsui FH, Cavalcanti AN. Effect of remaining dentine structure and thermalmechanical aging on the fracture resistance of bovine roots with different post and core systems. Int Endod J 2008;41(11):969-76.

36. Chawla K. Composite materials: science and engineering. 2nd ed. New York: Springer Ed; 1998.

37. Sidoli G, King P, Setchell D. An in vitro evaluation of a carbon fiber - based post and core system. J Prosthet Dent. 1997;78(1):5-9.

38. Lamas C, Alvarado S, Pari R, Poste Anatómico Preformado, Odontol. Sanmarquina, 2009;12(1):33-5.

39. Cury A, Goriacci C, de Lima Navarro M, Carvalho R, Sadek F, Tay F, Ferrari M. Effect of hygroscopic expansion on the pushout resisteance of glass ionomer-based cements used for the luting of glass fiber posts. Operative Dent 2006;32(6):537-40.

40. Dean JP, Jeansonne BG, Sarkar $N$. In vitro evaluation of a carbon fiber post. J Endod. 1998; 24(12):807-10.

41. Zogheib LV, Pereira JR, do Valle AL, de Oliveira JA, Pegoraro LF. Fracture resistance of weakened roots restored with composite resin and glass fiber post. Braz Dent J. 2008;19(4):329-33.
42. Fredriksson M, Astback J, Pamenius $\mathrm{M}$, Arvdison K. A retrospective study of 236 patients with teeth restored by carbon fiber-reinforced epoxy resin posts. J Prosthet Dent. 1998;80(2):151-7.

43. Ferrari M, Vichi A, Garcia-Godoy F. Clinical evaluation of fiberresin forced epoxy-resin posts and cast post-an-care. Am J Dent. 2000;13(Spec No):15B-18B.

44. Lassila LP, Tanner J, Le Bell AM, Narva K, Vallittu PK. Flexural properties of fiber reinforced root canal posts. Dent Mater. 2004;20(1):29-36.

45. Eskitascioglu G, Belli S, Kalkan M. Evaluation of two post core system using two different methods (fracture strength test and a finite elemental stress analysis). J Endod. 2002;28(9):629-33

46. Schwartz R. Fundamentos de odontología - operatoria un logro contemporáneo. 1a edición. California: Ed Actualidades Médico Odontológicas Latinoamericanas; 2000

47. Schwartz R, Robbins J. Post placement and restoration of endodontically treated teeth: a literature review. J Endod, 2004;30(5):289-301.

48. D’Arcangelo C, Canella M, De Angelis F, D'Amario M. The effect of resin cement film thickness on the pullout strength of a fiberreinforced post system. J Prosthet Dent. 2007;98(3):193-8.

49. Goracci C, Fabianelli A, Sadek FT, Papacchini F, Tay FR, Ferrari $M$. The contribution of friction to the dislocation resistance of bonded fiber posts. J Endod. 2005;31(8):608-12. 